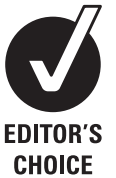

CHOICE
- Additional tables are published online only. To view these files please visit the journal online (http://heart.bmj. com)

${ }^{1}$ Department of Cardiology, Mazankowski Alberta Heart Institute, University of Alberta Edmonton, Canada

${ }^{2} T$ The Canadian VIGOUR Center Edmonton, Alberta, Canada ${ }^{3}$ Division of General Internal Medicine, University of Alberta, Edmonton, Canada

\section{Correspondence to}

Dr Roopinder K Sandhu, University of Alberta, 2C2. 30z WMC, 8440-112 Street, Edmonton, Alberta T6G 2B7, Canada; rsandhu2@ualberta.ca

This study is based, in part, on de-identified data provided by Alberta Health and Wellness through the Alberta Cardiac Access Collaborative; however, the interpretation and conclusions contained herein do not necessarily represent the views of the government of Alberta nor Alberta Health and Wellness.

Accepted 28 September 2011 Published Online First 10 November 2011

\title{
Risk stratification schemes, anticoagulation use and outcomes: the risk-treatment paradox in patients with newly diagnosed non-valvular atrial fibrillation
}

\author{
Roopinder K Sandhu, ${ }^{1}$ Jeffrey A Bakal, ${ }^{2}$ Justin A Ezekowitz, ${ }^{1,2}$ Finlay A McAlister ${ }^{3}$
}

\begin{abstract}
Objective To examine whether warfarin use and outcomes differ across $\mathrm{CHADS}_{2}$ and $\mathrm{CHA}_{2} \mathrm{DS}_{2}$-VASc risk strata for non-valvular atrial fibrillation (NVAF).

Design Population-based cohort study using linked administrative databases in Alberta, Canada.

Setting Inpatient and outpatient.

Patients 42834 consecutive patients $\geq 20$ years of age with newly diagnosed NVAF.
\end{abstract}

Main outcome measures Cerebrovascular events and/ or mortality in the first year after diagnosis.

Results Of 42834 NVAF patients, $22.7 \%$ were low risk on the $\mathrm{CHADS}_{2}$ risk score (0), $27.5 \%$ were intermediate risk (1), and $49.8 \%$ were high risk ( $\geq 2)$. The $\mathrm{CHA}_{2} \mathrm{DS}_{2}$ VASc risk score reclassified 16722 patients such that $7.8 \%$ were defined low risk, $13.8 \%$ intermediate risk and $78.4 \%$ high risk. Of the elderly cohort ( $\geq 65$ years) with definite NVAF visits (at least two encounters 30 days apart, $n=8780$ ), 49\% were taking warfarin within 90 days of diagnosis. Warfarin use did not differ across risk strata using either the $\mathrm{CHADS}_{2}$ ( $p$ for trend $=0.85$ ) or $\mathrm{CHA}_{2} \mathrm{DS}_{2}$-VASC $(\mathrm{p}=0.35)$. In multivariable adjusted analyses, warfarin use was associated with substantially lower rates of death or cerebrovascular events for patients with $\mathrm{CHADS}_{2}$ scores of 1 (OR 0.52, 95\% Cl 0.41 to 0.67 ) or $\geq 2$ (OR $0.61,95 \% \mathrm{Cl} 0.53$ to 0.71$)$, or $\mathrm{CHA}_{2} \mathrm{DS}_{2}$-VASc scores of $\geq 2$ (OR $0.60,95 \% \mathrm{Cl} 0.53$ to 0.68 ).

Conclusions In elderly patients with NVAF and elevated $\mathrm{CHADS}_{2}$ or $\mathrm{CHA}_{2} \mathrm{DS}_{2}$-VASC scores, warfarin users exhibited lower rates of cerebrovascular events and mortality. However, warfarin use did not differ across risk strata, another example of the risk-treatment paradox in cardiovascular disease.

Atrial fibrillation (AF) is the most common cardiac rhythm disorder and its prevalence continues to rise. ${ }^{1}{ }^{2}$ Stroke prevention in patients with AF is important in reducing morbidity, mortality and associated healthcare costs. ${ }^{3-6}$ Despite the plethora of randomised trials and observational studies establishing the efficacy, cost-effectiveness and relative safety of oral anticoagulation in patients with $\mathrm{AF}$, it remains under-utilised. ${ }^{7} 8$

Current guidelines for the management of $\mathrm{AF}$ provide recommendations for anticoagulation based on the $\mathrm{CHADS}_{2}$ (Congestive heart failure, Hypertension, Age $\geq 75$ years, Diabetes mellitus, and prior Stroke or transient ischaemic attack) scheme for stratifying stroke risk. ${ }^{9}$ Recently, the $\mathrm{CHA}_{2} \mathrm{DS}_{2}$-VASc (Congestive heart failure,
Hypertension, Age $\geq 75$ years, Diabetes mellitus, prior Stroke or transient ischaemic attack-Vascular disease, age 65-74, female sex) has been proposed as a modification of the $\mathrm{CHADS}_{2}$ score to reduce the proportion of patients classified in the 'intermediate' risk category. ${ }^{10}$ In a Danish study which used prescribing data to define co-morbidities, $\mathrm{CHA}_{2} \mathrm{DS}_{2}$-VASc score predicted the risk of thromboembolism and/or death better than the $\mathrm{CHADS}_{2}$ score in hospitalised patients.

Studies in patients hospitalised with a history of AF have found low rates of warfarin use, even among patients with high $\mathrm{CHADS}_{2}$ scores. ${ }^{8} \mathrm{~A}$ recent retrospective cohort study using the US MarketScan Database ${ }^{11}$ reported that $43 \%$ of eligible AF patients received warfarin with no appreciable difference in utilisation rates across $\mathrm{CHADS}_{2}$ categories for patients with prevalent or incident diagnoses of AF. Similarly, the Euro Heart Survey on Atrial Fibrillation reported that only about half of their AF patients received warfarin with no significant association between $\mathrm{CHADS}_{2}$ score and likelihood of warfarin prescription. ${ }^{12}$ However, these studies examined prevalent AF cases and limited data exists on warfarin use and outcomes for patients newly diagnosed with nonvalvular atrial fibrillation (NVAF) across risk categories. We also recently demonstrated that the characteristics, treatments and outcomes for newly diagnosed AF patients differed substantially depending on locale of incident diagnosis and, although most AF studies focus only on hospitalised patients, $48 \%$ of all AF patients are initially detected outside of hospital. ${ }^{13}$ Therefore, we designed this study to examine whether warfarin prescribing differed across absolute risk strata using the $\mathrm{CHADS}_{2}$ and $\mathrm{CHA}_{2} \mathrm{DS}_{2}$-VASc scores for patients with NVAF newly diagnosed in any setting.

\section{METHODS}

\section{Study population}

The University of Alberta Health Research Ethics Board approved this study. We have previously published detailed methods. ${ }^{13}$ Briefly, we obtained data by linking five administrative databases in a single-payer healthcare system with universal access and $100 \%$ capture of all interactions with the healthcare system: (1) the Discharge Abstract Database records dates for all admissions to acute care facilities with the most responsible diagnosis and up to 25 other diagnoses or co-morbidities; (2) 
the Ambulatory Care Database tracks all visits to hospital-based physicians' offices or emergency departments (ED), and allows for coding of up to six conditions for the years 2000-02 and 10 conditions from 2003 to 2005; (3) the Physician Claims Database tracks all physician claims for outpatient services and includes up to three diagnoses per encounter; (4) the Alberta Health Care Insurance Registry tracks the vital status of all 3.5 million Albertans, and the (5) Alberta Blue Cross Drug Database tracks all medication dispensations for individuals 65 years of age and older.

\section{Data elements and variable definitions}

We identified patients $\geq 20$ years with a new diagnosis of $\mathrm{AF}$ (ICD-9-CM 427.3 or ICD-10 code I48) ${ }^{14}$ between 1 January 2000 and 31 December 2005 in any of the datasets listed above, and excluded those with mitral or aortic disease (ICD-9 CM 394-396, 424.0, 424.1 or ICD-10 I05, I06, I34, I35) or valve surgery (ICD-9 CM 35.11, 35.12, 35, 24 and CCI code HU and $\mathrm{HV}$ ) up to and including the incident date of the AF diagnosis to define our cohort of patients with newly diagnosed NVAF. The accuracy of these ICD codes for AF in these databases has been previously validated against ECG data and chart audit in Alberta, ${ }^{14}$ with very high specificity (95-99.9\%) and positive predictive value (91-99\%), and reasonable sensitivity (58-96\%).

Patients were defined as having definite NVAF if they had at least two encounters with the healthcare system with a diagnosis of NVAF within a year, but more than 30 days apart; for the analyses relating medication use to 1 year outcomes, we only included patients who survived at least 90 days after initial AF diagnosis (thereby mitigating the risk of immortal time bias since all patients analysed had the opportunity to receive a prescription post-NVAF diagnosis).

\section{Co-morbidities and thromboembolic risk}

Co-morbidities were defined as those present and known at the time of the incident NVAF diagnosis and were based on diagnoses entered at that index visit and all other contacts with the healthcare system in the 12 months preceding the index NVAF visit (as previously validated in Alberta datasets). ${ }^{14-16}$

$\mathrm{CHADS}_{2}$ and $\mathrm{CHA}_{2} \mathrm{DS}_{2}$-VASc score ${ }^{9}{ }^{10}$ were determined by using age and co-morbidities noted at time of index NVAF diagnosis, and interrogating all healthcare visits in the 12 months prior to NVAF diagnosis for relevant co-morbidities. We have previously demonstrated the value of including diagnoses from healthcare encounters prior to the index NVAF visit in constructing a complete co-morbidity picture for each patient-almost one quarter of patients diagnosed with NVAF as outpatients had been hospitalised at least once in the prior year; the median number of outpatient visits for all patients in our NVAF cohort was 12 in the year prior to AF diagnosis. ${ }^{13}$ Patients were stratified using $\mathrm{CHADS}_{2}$ and $\mathrm{CHA}_{2} \mathrm{DS}_{2}$-VASc scores into low ( 0 points), intermediate (1 point) and high ( $\geq 2$ points) risk categories.

\section{Warfarin use}

Our definite NVAF cohort was linked to the Blue Cross Database to extract medication use for individuals 65 years of age. As such, we could not examine warfarin use in the subgroup with $\mathrm{CHA}_{2} \mathrm{DS}_{2}$-VASc risk score of 0 since all patients were older than 65 years. We excluded patients with prescriptions for warfarin filled prior to their first diagnostic code for NVAF. The use of aspirin could not be evaluated because over the counter medications are not tracked, and clopidogrel was prescribed in only 304 patients and therefore not considered further. We examined prescriptions within 90 days after initial diagnosis of NVAF as the median prescription interval in Alberta is 61 days.

\section{Outcomes}

The primary outcome of interest was cerebrovascular event and/ or mortality in the first year after incident NVAF diagnosis.

\section{Statistical analysis}

Results are presented as mean $\left(\mathrm{SD}^{17}\right)$ or median $\left(\mathrm{IOR}^{18}\right)$, compared using t-tests or Wilcoxon rank sum scores; counts and percentages were analysed using $\chi^{2}$ or Fisher's exact and Cochran-Armitage tests for trends. A logistic regression model was used with a stepwise variable selection (entry criteria $\mathrm{p}=0.10)$ to generate adjusted ORs and $95 \%$ CIs for a composite of cerebrovascular event or mortality with and without warfarin. The model comparing warfarin use/non-use and outcomes included patients over 65 years with two healthcare encounters 30 days apart but within a year, and who did not die within 90 days of initial diagnosis. Multivariate analysis was adjusted for age and all co-morbidities and location of diagnosis. All tests were two-sided, with the level of significance set at $p<0.05$ unless otherwise indicated, and performed using SAS V.9.2.

\section{RESULTS}

Of the 42834 patients with newly diagnosed NVAF, 9732 $(22.7 \%)$ were defined as low risk (score 0), 11787 (27.5\%) as intermediate risk (score 1), and 21315 (49.8\%) as high risk (score $\geq 2$ ) using the $\mathrm{CHADS}_{2}$ scheme (table 1). Use of the $\mathrm{CHA}_{2} \mathrm{DS}_{2}$ VASc scheme resulted in 16722 patients being reclassified such that $3320(7.8 \%)$ were defined as low risk (score 0), 5915 (13.8\%) as intermediate risk (score 1), and 33599 (78.4\%) as high risk.

One year rates of mortality and the composite of cerebrovascular events or mortality differed significantly $(p<0.001)$ across both $\mathrm{CHADS}_{2}$ and $\mathrm{CHA}_{2} \mathrm{DS}_{2}$-VASc risk strata (figure 1). There was only moderate agreement between $\mathrm{CHADS}_{2}$ and $\mathrm{CHA}_{2} \mathrm{DS}_{2}$-VASC scores for outcomes when both were broken down into three risk strata: $\kappa=0.44$ ( $95 \%$ CI 0.43 to 0.44 ).

Of the 8780 elderly patients with definite NVAF, 936 (10.7\%) were defined as low risk, 2393 (27.3\%) as intermediate risk, and $5481(62.1 \%)$ as high risk (score $\geq 2$ ) on the $\mathrm{CHADS}_{2}$ criteria. When the $\mathrm{CHA}_{2} \mathrm{DS}_{2}$-VASc risk scheme was applied to the elderly subgroup, 395 (4.5\%) were defined as intermediate risk and 8385 (95.5\%) as high risk. Across $\mathrm{CHADS}_{2}$ and $\mathrm{CHA}_{2} \mathrm{DS}_{2}$-VASc risk categories there were significant differences in use of cardiac medications (S1), except for warfarin. Overall, 4304 (49.0\%) of our elderly cohort with definite NVAF were taking warfarin within 90 days of initial diagnosis, and warfarin use did not differ across $\mathrm{CHADS}_{2}$ strata ( 1 1, figure 2, p for trend=0.85) or $\mathrm{CHA}_{2} \mathrm{DS}_{2}$-VASC strata ( $\mathrm{S} 1$, figure $2, \mathrm{p}=0.35$ ). Excluding patients with potential contraindications to warfarin (prior history of bleeding, anaemia or cancer) did not change our findings: warfarin usage was $51.0 \%$ in $\mathrm{CHADS}_{2}$ score $0,50.5 \%$ in $\mathrm{CHADS}_{2}$ score 1 , and $52.3 \%$ in $\mathrm{CHADS}_{2}$ score $\geq 2$ ( $\mathrm{p}$ for trend $=0.35$ ), and $51.6 \%$ in $\mathrm{CHA}_{2} \mathrm{DS}_{2}$-VASC score 1 versus $54.1 \%$ in $\mathrm{CHA}_{2} \mathrm{DS}_{2}$-VASC score $\geq 2(\mathrm{p}=0.41)$. On multivariate analysis, factors associated with reduced likelihood of being prescribed warfarin included initial diagnosis in an outpatient clinic (OR $0.51,95 \%$ CI 0.29 to 0.90 ), history of dementia (OR $0.55,95 \% \mathrm{CI}$ 0.40 to 0.76 ), gastrointestinal bleed (OR $0.55,95 \%$ CI 0.38 to 0.80 ) or anaemia (OR $0.74,95 \%$ CI 0.60 to 0.91 ). Use of various cardiac medications was associated with increased likelihood of using warfarin: ACE inhibitors/angiotensin receptor blockers (OR 1.49, 95\% CI 1.23 to 1.80); $\beta$-blockers (OR 2.37, 95\% CI 
Table 1 Baseline characteristics, stratified by $\mathrm{CHADS}_{2}$ stroke risk categories

\begin{tabular}{|c|c|c|c|c|}
\hline \multirow[b]{2}{*}{ Characteristics } & \multicolumn{4}{|c|}{$\mathrm{CHADS}_{2}$ risk category } \\
\hline & Total $(n=42834)$ & Low $(n=9732)$ & Intermediate $(n=11787)$ & High $(n=21315)$ \\
\hline Median age (IQR) & $73(81-107)$ & $57(45-67)$ & $71(62-78)$ & $79(73-85)$ \\
\hline Heart failure & $11039(25.8)$ & $0(0)$ & $1311(11.1)$ & $9728(45.6)$ \\
\hline Hypertension & $23125(54.0)$ & $0(0)$ & $5943(50.4)$ & $17182(80.6)$ \\
\hline Diabetes & $7891(18.4)$ & $0(0)$ & $942(8.0)$ & $6949(32.6)$ \\
\hline Ischaemic heart disease & $15922(37.2)$ & $1778(18.3)$ & $3861(32.8)$ & $10299(48.32)$ \\
\hline Peripheral artery disease & $3430(8.0)$ & $200(2.1)$ & $618(5.2)$ & $2612(12.3)$ \\
\hline Age $65-74$ & $11159(26.1)$ & $2856(29.4)$ & $4319(36.6)$ & 3984 (18.7) \\
\hline Female & $20301(47.4)$ & $3971(40.8)$ & $5210(44.2)$ & $11120(52.2)$ \\
\hline \multicolumn{5}{|l|}{ Other co-morbidities } \\
\hline Chronic kidney disease & $3265(7.6)$ & 1778 (18.3) & 3861 (32.8) & $10299(48.3)$ \\
\hline Gastrointestinal bleed & $2192(5.1)$ & $244(2.5)$ & $478(4.1)$ & $1470(6.9)$ \\
\hline Rural residence & $8445(19.7)$ & $1827(18.8)$ & $2427(20.6)$ & 4191 (19.7) \\
\hline Diagnosed by specialist & 17750 (41.4) & $4047(41.6)$ & $4741(40.2)$ & $8962(42.1)$ \\
\hline
\end{tabular}

Values are $\mathrm{n}(\%)$.

Specialist defined as cardiologist or internist.

CVA, cerebral vascular attack; TIA, transient ischaemic attack.

1.96 to 2.87 ); diuretics (OR $1.75,95 \%$ CI 1.44 to 2.12 ); calcium channel blockers (OR 2.56, 95\% CI 2.02 to 3.24); digoxin (OR 2.33, $95 \%$ CI 1.92 to 2.82 ); statins (OR $1.44,95 \%$ CI 1.10 to 1.87); and anti-arrhythmic medications (OR 2.42, 95\% CI 1.87 to 3.13$)$.

In multivariate analyses adjusting for location of diagnosis, age, sex, the variables in table 1 , and cardiac medications, warfarin use was associated with substantially lower rates of cerebrovascular event and/or mortality in the first year after NVAF diagnosis in those patients with baseline $\mathrm{CHADS}_{2}$ scores of 1 or greater, or $\mathrm{CHA}_{2} \mathrm{DS}_{2}$-VASc scores of 2 or greater (table 2). Among patients 75 years or older, warfarin use was associated with a $42 \%$ lower rate of cerebrovascular event and/or mortality (S2). In this subgroup of patients 75 years or older, the association between warfarin use and lower event rates was similar regardless of the stroke risk score.

\section{DISCUSSION}

In this population-based study of newly diagnosed NVAF, in which approximately half the cohort were outpatients, there
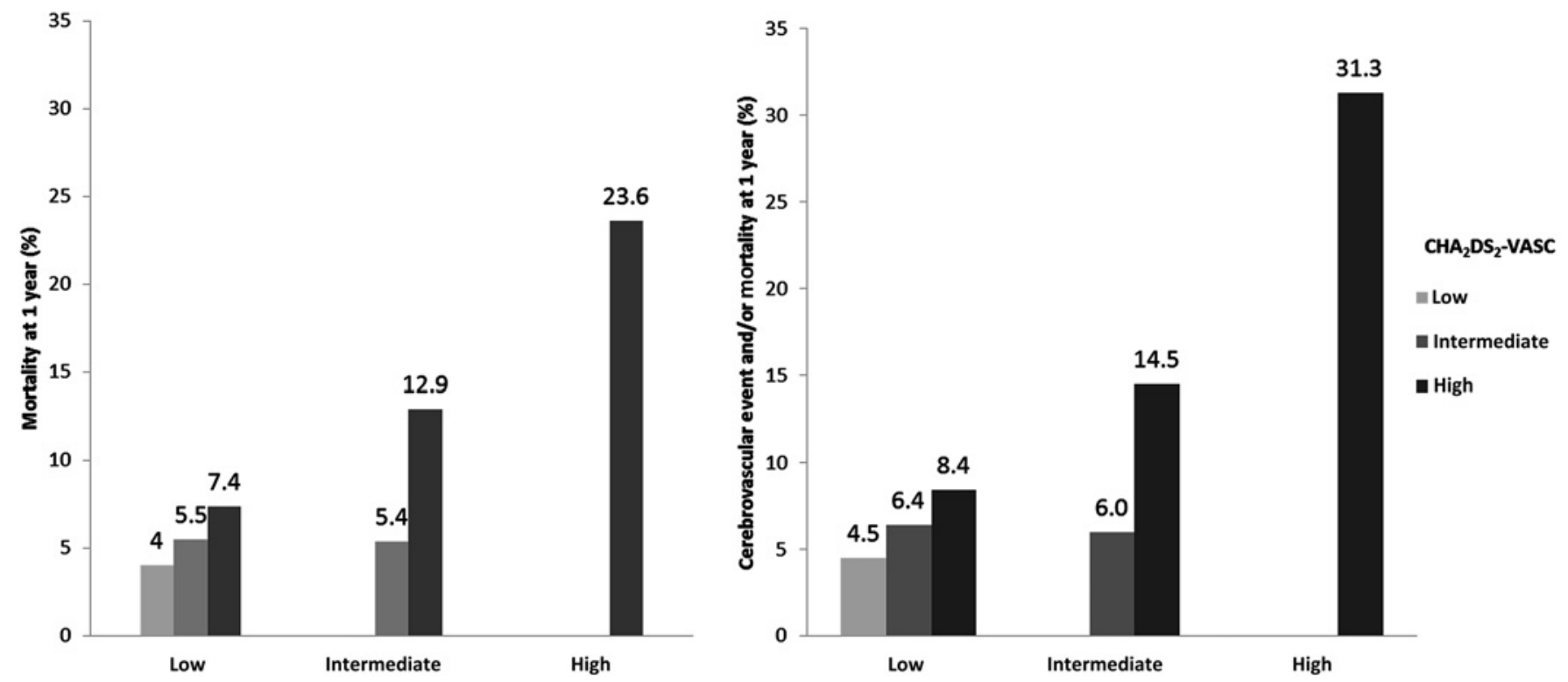

\section{$\mathrm{CHADS}_{2}$ risk categories}

Figure 1 One year rates of mortality or the composite of cerebrovascular event or mortality, stratified by $\mathrm{CHA}_{2} \mathrm{DS}_{2}-\mathrm{VASc}_{\mathrm{S}}$ and $\mathrm{CHADS}_{2}$ risk category. 


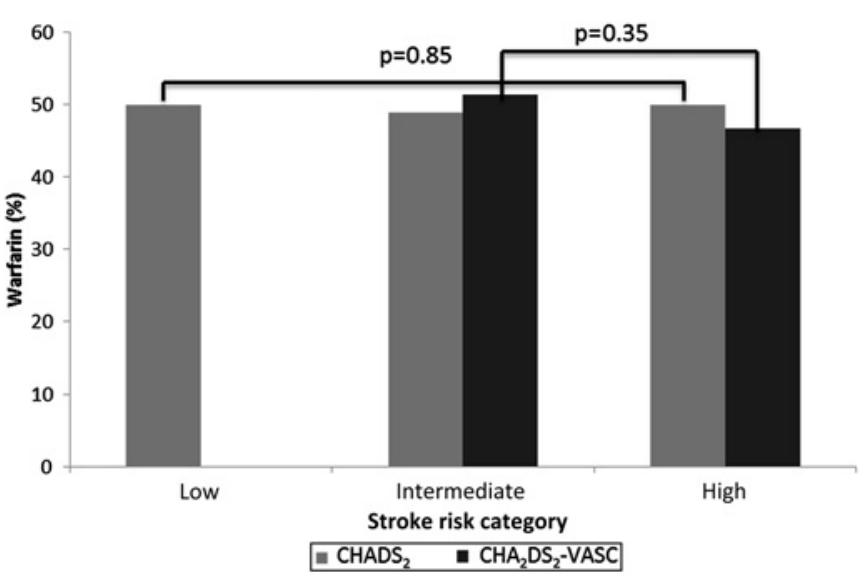

Figure 2 Rates of warfarin use by $\mathrm{CHADS}_{2}$ and $\mathrm{CHA}_{2} \mathrm{DS}_{2}$-VASc risk categories.

was only moderate agreement between the $\mathrm{CHADS}_{2}$ and $\mathrm{CHA}_{2} \mathrm{DS}_{2}$-VASc risk scores, with $39 \%$ of patients being reclassified into a higher stratum using the $\mathrm{CHA}_{2} \mathrm{DS}_{2}$-VASc scheme. our data demonstrates a clear relationship between increasing $\mathrm{CHADS}_{2}$ and $\mathrm{CHA}_{2} \mathrm{DS}_{2}$-VASc scores and higher rates of cerebrovascular events and/or mortality mirror results reported from the General Practice Research Database in the UK, ${ }^{19}$ the Danish national register, ${ }^{20}$ and the Anticoagulation and Risk Factors in Atrial Fibrillation (ATRIA) studies. ${ }^{21}$ On the other hand, some have argued that current stroke risk stratification schemes in AF have weak predictive power, ${ }^{22}$ and Keogh et al reported a pooled c statistic of only 0.60 (95\% CI 0.43 to 0.72 ) for ischaemic stroke with the $\mathrm{CHADS}_{2}$ score. $^{23}$

Although the under-utilisation of warfarin in patients with NVAF that we found is not a novel finding, ${ }^{8}$ and multiple potential explanations have been offered in the past, ${ }^{7} 22$ our finding that warfarin is prescribed at similar rates among newly diagnosed NVAF patients with low absolute stroke risk as among those at high stroke risk is contrary to guideline recommendations. Our results echo prior studies from the USA ${ }^{11}$ and Europe $^{12}$ which did not find a significant gradient in warfarin use across risk strata. However, our study extends the evidence base by examining a broader spectrum of patients with incident NVAF diagnosed across all healthcare settings, while earlier studies included a mix of prevalent and incident cases largely from inpatient settings. We believe that this lack of a positive gradient in warfarin use by $\mathrm{CHADS}_{2}$ and $\mathrm{CHA}_{2} \mathrm{DS}_{2}$-VASc scores is a manifestation of the 'risk-treatment paradox' that has been described for other cardiac conditions, ${ }^{24}$ in that proven efficacious therapy is not more likely to be prescribed to higher risk patients. Of course, patients at higher absolute risk of stroke may also have higher bleeding risks ${ }^{25}$ and thus physicians may be weighing potential benefits and risks in each patient and tailoring therapy appropriately-although our analysis excluding those patients with potential contraindications to warfarin also failed to demonstrate a gradient across $\mathrm{CHADS}_{2}$ or $\mathrm{CHA}_{2} \mathrm{DS}_{2}$-VASc strata. It should be acknowledged that administrative databases do not capture all the subtleties of clinical care (such as functional status) which may account for this apparent risk-treatment paradox. ${ }^{26}$

The substantial use of warfarin in patients at low absolute stroke risk is a concern given the bleeding risks of warfarin; indeed, analyses of the ATRIA study data suggest that warfarin is associated with apparent harm rather than benefit in patients with $\mathrm{CHADS}_{2}$ scores of $0 .{ }^{21}$ Additionally, Olesen et al demonstrated a negative net clinical benefit of oral anticoagulation with a $\mathrm{CHA}_{2} \mathrm{DS}_{2}$-VASc score 0 in a large Danish cohort regardless of bleeding risk stratification score. ${ }^{27} \mathrm{~A}$ recent decision analytic model demonstrated that aspirin is more beneficial than warfarin when the baseline stroke risk is $<1.7 \%$ per year. ${ }^{28}$

Although current guidelines recommend either antiplatelet agents or anticoagulation use for patients at intermediate stroke risk, we found that warfarin users with a $\mathrm{CHADS}_{2}$ score of 1 exhibited substantially lower rates of cerebrovascular events and/or mortality than warfarin non-users. Similarly, the ATRIA study suggested a net clinical benefit from warfarin for those patients with a $\mathrm{CHADS}_{2}$ score of $1 .^{21}$

Within a given $\mathrm{CHADS}_{2}$ risk stratum, we found the 1 year event rates of mortality and composite endpoint were higher with increasing $\mathrm{CHA}_{2} \mathrm{DS}_{2}$-VASc risk scores. ${ }^{9} 29$ Our outcome rates are higher than previously reported studies such as ATRIA, as nearly $50 \%$ of our cohort were initially inpatients and we evaluated patients with newly diagnosed NVAF, while the ATRIA population consisted of prevalent warfarin users and excluded patients with prior major bleeding. A retrospective study in Scotland demonstrated a one-year mortality after AF diagnosis as high as $33 \%$ for hospitalised patients. ${ }^{3}$

There are some potential limitations to our study. Administrative databases can be subject to misclassification, however the ICD codes we used have been previously validated. ${ }^{14}$ Our comorbidity data likely underestimates true co-morbidity burden (since administrative codes have excellent specificity but poorer sensitivity for several elements of the $\mathrm{CHADS}_{2}$ and $\mathrm{CHA}_{2} \mathrm{DS}_{2-}$ VASc scores), although the advantage of our dataset is that it captures all physician-entered diagnoses and thus we have accurately captured the co-morbidity burden recognised by each patient's treating physician. We were unable to determine the number of patients who had successfully undergone cardioversion after initial NVAF diagnosis in our dataset as we did not

Table 2 One-year risk of cerebrovascular event, mortality or the composite of cerebrovascular event or mortality in warfarin users and non-users, stratified by stroke risk category at time of non-valvular atrial fibrillation diagnosis

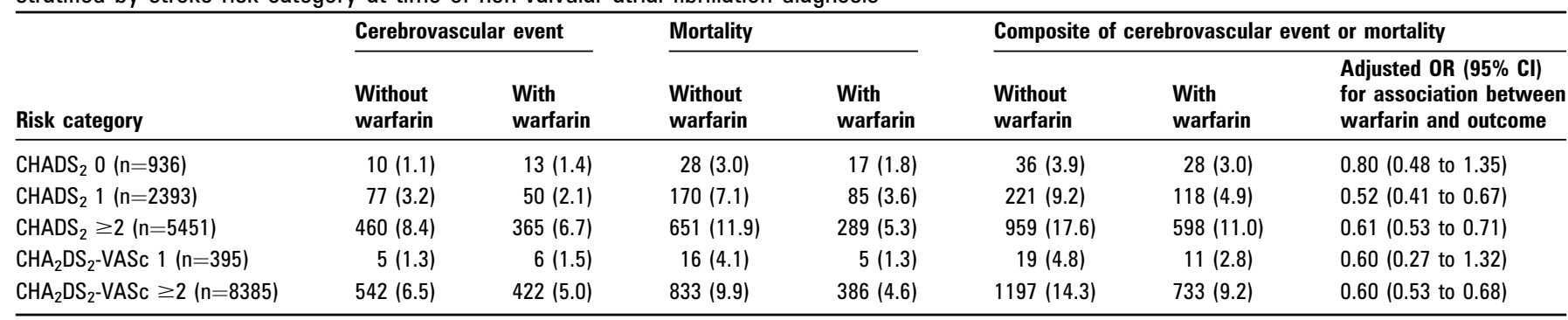

Values are $\mathrm{n}(\%)$ unless otherwise indicated.

Multivariable model adjusted for co-morbidities, medications and location. 
have access to ECG data; however, the fact that less than one fifth of our patients were prescribed antiarrhythmic therapy would suggest that cardioversion (or at least chemical cardioversion) was infrequently deployed in our population. Our medication data is based on pharmacy dispensations and thus is a function of both physician intent to treat and patient adherence to treatment recommendations. Also, the Blue Cross Database only captures prescriptions in patients older than 65 and thus usage rates in younger patients (ie, those with $\mathrm{CHA}_{2} \mathrm{DS}_{2}$-VASc risk scores of 0 ) could not be ascertained. Although we examined outcomes in those patients using warfarin, we did not have access to their international normalised ratio (INR), therefore time in the target range could not be calculated. We were unable to calculate a bleeding risk stratification score (such as HAS-BLED) as several key components required for score calculation such as liver function tests, serum creatinine, INR, and information on drug or alcohol use is not available in our databases. Lastly, this is an observational study and inferences about causation between prescribed therapies and outcomes should be tempered due to the inability to adjust for unmeasured confounders.

In conclusion, we found no difference in warfarin use across stroke risk strata as defined by either the $\mathrm{CHADS}_{2}$ or the $\mathrm{CHA}_{2} \mathrm{DS}_{2}$-VASc score. Thus, warfarin is over-utilised in low risk patients and under-utilised in high risk patients. This emphasises the importance of developing and implementing strategies to improve antithrombotic prescribing practices in patients with NVAF. $^{30}$ Although the introduction of newer agents such as dabigatran will change the risk/benefit ratio of antithrombotic therapy for $\mathrm{NVAF}^{28}$ appropriately tailoring therapy on the basis of each individual's stroke risk will remain the key issue even with the newer antithrombotic agents.

Funding JAE is supported by the Canadian Institutes of Health Research and Alberta Innovates-Health Solutions (AlHS). FAM is supported by AIHS.

Competing interests None.

Ethics approval Alberta Health Research Ethics Board.

Contributors RKS designed and wrote the research plan, analysed data, and drafted and revised the manuscript. She is guarantor. JAB performed statistical analyses and revised the manuscript. JAE designed the research plan, analysed data, and drafted and revised the manuscript. FAM designed the research plan, analysed data, and drafted and revised the manuscript.

Provenance and peer review Not commissioned; externally peer reviewed.

\section{REFERENCES}

1. Wolf PA, Benjamin EJ, Belanger AJ, et al. Secular trends in the prevalence of atrial fibrillation: the Framingham Study. Am Heart J 1996;131:790-5.

2. Miyasaka Y, Barnes ME, Gersh BJ, et al. Secular trends in incidence of atrial fibrillation in Olmsted County, Minnesota, 1980 to 2000, and implications on the projections for future prevalence. Circulation 2006;114:119-25.

3. Stewart S, Hart CL, Hole DJ, et al. A population-based study of the long-term risks associated with atrial fibrillation: 20-year follow-up of the Renfrew/Paisley study. Am J Med 2002:113:359-64.
4. Benjamin EJ, Wolf PA, D'Agostino RB, et al. Impact of atrial fibrillation on the risk of death: the Framingham Heart Study. Circulation 1998;98:946-52.

5. Wolf PA, Mitchell JB, Baker CS, et al. Impact of atrial fibrillation on mortality, stroke, and medical costs. Arch Intern Med 1998;158:229-34.

6. Coyne KS, Paramore C, Grandy S, et al. Assessing the direct costs of treating nonvalvular atrial fibrillation in the United States. Value Health 2006;9:348-56.

7. Bungard TJ, Ghali WA, Teo KK, et al. Why do patients with atrial fibrillation not receive warfarin? Arch Intern Med 2000;160:41-6.

8. Ogilvie IM, Newton N, Welner SA, et al. Underuse of oral anticoagulants in atrial fibrillation: a systematic review. Am J Med 2010;123:638-45 e4.

9. Gage BF, Waterman $A D$, Shannon W, et al. Validation of clinical classification schemes for predicting stroke: results from the National Registry of Atrial Fibrillation. JAMA 2001;285:2864-70.

10. Lip GY, Nieuwlaat R, Pisters R, et al. Refining clinical risk stratification for predicting stroke and thromboembolism in atrial fibrillation using a novel risk factor-based approach: the euro heart survey on atrial fibrillation. Chest 2010;137:263-72.

11. Zimetbaum PJ, Thosani A, Yu HT, et al. Are atrial fibrillation patients receiving warfarin in accordance with stroke risk? Am J Med 2010;123:446-53.

12. Nieuwlaat R, Capucci A, Lip GY, et al. Antithrombotic treatment in real-life atrial fibrillation patients: a report from the Euro Heart Survey on Atrial Fibrillation. Eur Heart J 2006;27:3018-26.

13. Sandhu R, Bakal JA, Ezekowitz JA, et al. The epidemiology of atrial fibrillation in adults depends on locale of diagnosis. Am Heart J 2011;161:986-92.

14. Kokotailo RA, Hill MD. Coding of stroke and stroke risk factors using international classification of diseases, revisions 9 and 10. Stroke 2005;36:1776-81.

15. Quan H, Parsons GA, Ghali WA. Validity of information on comorbidity derived rom ICD-9-CCM administrative data. Med Care 2002;40:675-85.

16. Quan H, Khan N, Hemmelgarn BR, et al. Validation of a case definition to define hypertension using administrative data. Hypertension 2009;54:1423-8.

17. Alter $\mathbf{P}$, Waldhans S, Plachta E, et al. Complications of implantable cardioverter defibrillator therapy in 440 consecutive patients. Pacing Clin Electrophysiol 2005;28:926-32

18. Levey AS, Stevens LA, Schmid CH, et al. A new equation to estimate glomerular filtration rate. Ann Intern Med 2009:150:604-12.

19. Van Staa TP, Setakis E, Di Tanna GL, et al. A comparison of risk stratification schemes for stroke in 79,884 atrial fibrillation patients in general practice. J Thromb Haemost 2011;9:39-48.

20. Olesen JB, Lip GY, Hansen ML, et al. Validation of risk stratification schemes for predicting stroke and thromboembolism in patients with atrial fibrillation: nationwide cohort study. BMJ 2011;342:d124.

21. Singer DE, Chang $Y$, Fang MC, et al. The net clinical benefit of warfarin anticoagulation in atrial fibrillation. Ann Intern Med 2009:151:297-305.

22. Nieuwlaat R, Connolly SJ. Stroke prevention in atrial fibrillation: better use of anticoagulation and new agents will lead to improved outcomes. Heart 2009:95:95-7.

23. Keogh C, Wallace E, Dillon C, et al. Validation of the CHADS2 clinical prediction rule to predict ischaemic stroke. A systematic review and meta-analysis. Thromb Haemost 2011;106:528-38.

24. Spertus JA, Furman Ml. Translating evidence into practice: are we neglecting the neediest? Arch Intern Med 2007:167:987-8.

25. Hughes M, Lip GY. Risk factors for anticoagulation-related bleeding complications in patients with atrial fibrillation: a systematic review. OJM 2007;100:599-607.

26. McAlister FA, Oreopoulos A, Norris CM, et al. Exploring the treatment-risk paradox in coronary disease. Arch Intern Med 2007;167:1019-25

27. Olesen JB, Lip GY, Lindhardsen J, et al. Risks of thromboembolism and bleeding with thromboprophylaxis in patients with atrial fibrillation: a net clinical benefit analysis using a 'real world' nationwide cohort study. Thromb Haemost 2011; 106:739-49.

28. Eckman MH, Singer DE, Rosand J, et al. Moving the tipping point: the decision to anticoagulate patients with atrial fibrillation. Circ Cardiovasc Oual Outcomes 2011:4:14-21.

29. Go AS, Hylek EM, Chang $Y$, et al. Anticoagulation therapy for stroke prevention in atrial fibrillation: how well do randomized trials translate into clinical practice? JAMA 2003;290:2685-92.

30. McAlister FA. Atrial fibrillation, shared decision making, and the prevention of stroke. Stroke 2002;33:243-4. 